\title{
A RUNDOWN ON THE CHRONICLES OF ASTEROIDS INCLUDING CONTEMPORARY IDEAS
}

\author{
V. Julin Annapooranam \\ Department of Education \& Science, \\ DMI-St. Eugene University Chipata, Zambia. \\ V. Hari Krishna Prabu \\ Department of Education \& Science, \\ DMI-St. Eugene University Chipata, Zambia.
}

Dawn Jaison

Department of Education Science,

DMI St. John The Baptist, Lilongwe, Malawi

\begin{abstract}
Asteroids are lumps of rock or perhaps ice or may be solid metal that are left over of the solar system formation. Most of them are orbiting somewhere between the orbits of the planets Mars and Jupiter. Asteroids are different in their size, despite their size, asteroids can be dangerous. Many have hit the earth in the past and more are likely to crack our planet in the future. That's why scientists give more concentration in learning about asteroids and their population, orbits and physical characteristics.
\end{abstract}

Keywords: Asteroids, Trojan, Vesta, Ceres, Pallas

\section{INTRODUCTION}

Asteroids are found in large numbers between the planets Mars and Jupiter that they realized they were a new type of celestial object altogether. They called them asteroids. Asteroids are smaller bodies they orbit like planets around the sun but much smaller than the planets which are orbiting between the planets Mars and Jupiter, some asteroids are also found in the orbit path of some planets. Asteroids are the left overs of the creation of our solar system. Asteroids are also commonly referred to as planetoids or minor planets. The important thing that they have remained mostly unchanged for billions of years, a research in asteroid could reveal a lot about the solar system in the early stages. Meanwhile, they are not easy to spot because they often are made of dark material and are difficult to find against the darkness of outer space. There are several devoted surveys with the help of both spacecraft and Earth-based telescopes searching the space for asteroids. From this article, you can get the answers for the questions like, what are the compositions of asteroids? How are they named? How many are known? How are they classified? What is the largest and smallest asteroid? What are the effects by them? Let's get into the world of asteroids.

\section{ORIGIN OF ASTEROIDS}

Scientists sought to discover the missing planets began to sought the solar system and found our first asteroid. Asteroids are made up of small pieces of metal and rocks. Small particles collide with each other and if collision is not too hard, they will stick together. Since the asteroids are too close to Jupiter, they didn't continue to grow like all the other planets because Jupiter's gravity "stirs them up" and makes them move fast, so that when they collide with each other, they usually rebound or shatter instead of coupling. They were formed from gas and dust, similar to the other planets. They are remnants of the solar system formation. For an example, a carpenter carving a block of wood into a table and a chair. There will be chips of wood left over after the work is done. The table and the chair are the planets and the chips are the asteroids Bobrovnikoff,N.T. (1931). This is how the Asteroids were originated.

\section{A. COMPOSITION}

As asteroids composition are mainly determined by how close it is to the sun. The asteroids that are found near the sun are composed of carbon, with few amounts of nitrogen, hydrogen and oxygen, whereas the ones farther are composed of silicate rock. They are composed of oxygen and silicon, the two most abundant elements in the earth's crust. The metallic asteroids are made up of $80 \%$ iron and $20 \%$ mixture of few materials like nickel, iridium, palladium, platinum, gold, magnesium and other precious metals such as osmium, ruthenium and rhodium. There are a few that are composed of half metallic and half silicate. Other than metals, the elements that are needed for the water creation are present in asteroids and there is evidence that asteroids contain ice or water in their interiors and there's even indicators that water may have flowed on the surface of at least one asteroid. The idea is that when a smaller asteroid run into a bigger asteroid, the small asteroid could deliver a layer of ice into the bigger asteroid. The force of impact shortly turns the ice into water. And this water across the surface, creating the gullies. 


\section{International Journal of Engineering Applied Sciences and Technology, 2019 \\ Vol. 4, Issue 8, ISSN No. 2455-2143, Pages 89-93 \\ Published Online December 2019 in IJEAST (http://www.ijeast.com)}

\section{B. HOW ARE THEY NAMED?}

Several asteroids were discovered over the first half of the 19th century and classified as planets. William Horschol coined the name "Asteroid" in 1802, but other scientists allude to the new found objects as minor planets.

By 1851 there were 15 new asteroids and the process of naming modified to include numbers. For example, 99942 for Apophis. The International Astronomical Unions committee, on small body nomenclature is little less strict when it comes to naming the asteroids as against IAU naming committees. Suggestions are welcomed from the scientists and the public as well. A giant space rocks has been named after Mr. Spock (a cat named for the character of 'star trek' fame), rock musician Frank Zappa, beloved teachers such as Florida's Cynthia L.Roy, and more somber tributes such as the seven asteroids named for the space shuttle Columbia crew killed in 2003. Asteroids are also named for places and a variety of other things. Naming asteroids after pets is not anymore allowed. The Harvard Smithsonian centre for Astrophiphes keeps a fairly current list of asteroid names.

\section{NUMBERS OF ASTEROIDS (HOW MANY ARE THEY?)}

By the early 1990's in the Asteroid-II era, roughly 10000 asteroids had been discovered, only a tiny part of the total number of asteroids that are known to exist now. At that time asteroids were discovered by visually impinging photographic plates for light trails.

In the 1990's many of the major automated discovery surveys came online. By the year 2000 around the time of AsteroidsIII, 20,000 asteroids were known. By the year 2015, Asteroids-IV, there are around 700,000 asteroids with known orbits, disclosing many new information about the asteroid belt's charismatic past. About 100,000 asteroids measurements tells us about the surface composition, providing a wider view of the Asteroid belt as never before. With the explosion of asteroid physical measurement surveys, the Sloan Digital Sky Survey and the Wild-Field Infrared Survey Explorer it is timely to think about recent advances, leaving us well assured for the next generation of major examinations including Gaia and the large Synoptic Survey Telescope.

\section{POTENTIALLY HAZARDOUS ASTEROIDS}

Almost 1500 asteroids in this day and age are documented as having the capacity to cause serious harm and these are only the ones we know about. A potentially hazardous asteroid is an asteroid whose orbit comes nearer of about 0.05AU (about 7.5 million $\mathrm{km}$ ) to the earth and whose brightness implies a size, about $100 \mathrm{~m}$ across or more. Such objects would have highly destructive consequences if they were to collide with the earth. At the time of writing, the International Astronomical Union lists a total of 1439 potentially hazardous asteroids. This does not mean all these objects will eventually hit the earth, just that they have the potential to do so. Of course, this number represents only the PHA we know about. A latest survey by NASA's WISE Satellite proposed there are at least 4700 such objects. Although we are not in immediate risk, asteroids like this which are big enough to cause major havoc, especially in heavily populated areas, have hit earth every 200 to 300 years on average.

\section{E. CLASSIFICATIONS}

Asteroids are categorized by two ways. One is based on composition of asteroids and another one is based on their position.

\section{CLASSIFICATIONS BASED ON THEIR COMPOSITION}

Categorization by the composition tells us what the asteroid is composed of and this is connected to how far from the sun as asteroid formed. Some endured high temperature after they formed and partly melted with the iron sinking to the Centre and the forcing basaltic lava to the surface.

There are three basic types of asteroids, (i) C-Type (ii) MType (iii) S-Type.

\section{(i) C-Type Asteroids}

About $75 \%$ of the recognized asteroids are thought to be C-Type asteroids with no strong absorption features, which can be found in the outer region of the asteroid belt. Asteroids like this are most common type of asteroids and are as dark as a lump of 'coal' with an ordinary albedo of about 0.06 . They are thought to have a similar composition as the Sun like hydrogen or helium or other 'volatile' elements.

\section{(ii) S-Type Asteroids}

About 17\% asteroids are S-Type (Stony) asteroids which are considerably brighter with an ordinary albedo of 0.16 that means they are brighter than C-Type and they are normally found in the inner region of asteroid belt. (Asteroid Belt is the region of the solar system found between 2.0 and 3.3 AU from the sun, where the huge majority of the asteroids in the solar system have their orbits). These asteroids have an absorption feature because of the mineral called 'Olivine'. SType asteroids are slightly reddish in color.

\section{(iii)M-Type Asteroids}

About $8 \%$ of the Familiar asteroids are M-Type (metallic) asteroids. These asteroids are found in the middle of the asteroid belt and brighter than an average albedo of 0.19 and are mostly composed of metallic iron.

These are the three basic types of Asteroids and their composition, distance, Physical characteristics.

\section{CLASSIFICATIONS BASED ON THEIR POSITION IN SOLAR SYSTEM}

Asteroids are also categorized by their position in the solar system. They classified as follows (i) Main Asteroid Belt 


\section{International Journal of Engineering Applied Sciences and Technology, 2019 Vol. 4, Issue 8, ISSN No. 2455-2143, Pages 89-93 \\ Published Online December 2019 in IJEAST (http://www.ijeast.com)}

\section{(ii) Trojan (iii) Near - Earth Asteroids}

\section{(i) Main Asteroid Belt}

The majority of the familiar asteroids orbit within the asteroid belt, which is between the planets Mars and Jupiter, normally with not very elongated orbits. Derek C. Richardson, (2001). The belt is reckoned to contain 1.1 to 1.9 million asteroids, which are larger than $1 \mathrm{~km}$ in diameter and millions of smaller ones. During the earlier stages of the universe the gravity of the Jupiter stopped the formation of the planetary bodies in its region and this caused the remnants to collide with one another, imploding them into asteroids. Roig, F., (2007).

\section{(ii) Trojan}

The asteroids that share an orbit with a larger planet, yet do not collide with it since they rally around two special places in the orbit. There, the gravitational pull between the sun and the planets are balanced by a Trojan's tendency to otherwise fly out of the orbit, The Jupiter Trojans from the most significant population of Trojan asteroids. It is thought they are as numerous as the asteroids in the asteroid belt. There are Mars and Neptune Trojans, and in 2011 NASA reported the discovery of an Earth Trojan. Gradie, J., Veverka (1980).

\section{(iii) Near - Earth Asteroids}

These asteroids have orbits that pass nearby that of the Earth. Asteroids that actually cross the Earth's orbital path are known as Earth-crossers. As of June 19,2013, 10,003 near Earth asteroids are familiar and the number over $1 \mathrm{~km}$ in diameter is considered to be 861 with 1,409 categorized as potentially hazardous asteroids those that could cause a threat to Earth.

\section{F. SOME KNOWN ASTEROIDS}

Some hundred thousand asteroids have been discovered and given provisional designation so far. Thousands more are discovered each year. There are doubtlessly hundreds of thousands more that are too small to be seen from the earth. There are 26 familiar asteroids greater than $200 \mathrm{~km}$ in diameter. Our census of the largest ones is now fairly complete.

We probably are familiar of $99 \%$ of the asteroids greater than $100 \mathrm{~km}$ in diameter, of those in the 10 to $100 \mathrm{~km}$ range we have itemized about half. But we know very few of the smaller ones, there are considerably more than a million asteroids in the $1 \mathrm{~km}$ range. The total mass of all the asteroids is less than that of the moon.

\section{(i) 1 Ceres}

The greatest asteroid by far is 1 Ceres. It is $974 \mathrm{~km}$ in diameter and contains about $25 \%$ mass of all the asteroids, combined situated in asteroid belt between Mars and Jupiter and the only dwarf planet (means the spherical and travels in an orbit around the sun) located in the inner solar system. It was the first member of the asteroid belt to be discovered when Giuseppe Piazzi spotted it in 1801. Discovery of Ceres is an interesting story that it was found by an astronomer who was searching for a star. He thought he discovered a comet, but with the help of other astronomers resolute it was a planet. As more objects like this were discovered between Mars and Jupiter, scientists decided to be called an asteroid is the largest in the asteroid belt. Even though Ceres comprise $25 \%$ of the asteroid belt's total mass, tiny Pluto is still 14 times more massive. Ceres is named for the roman goddess of corn and harvests. The word cereal comes out of the same name.

Ceres is so much bigger, if the Earth were the size of the Nickel, Ceres would be about as big as a poppy seed. From a mean distance of 413 million $\mathrm{km}$, Ceres is $2.8 \mathrm{AU}$ away from the sun, it takes 1682 Earth days or 4.6 Earth years to make one trip around the sun. It completes one rotation around its axis every 9 hours. Ceres is also different from its neighbors, so that the scientists classified it as a dwarf planet in 2006. Meanwhile it does not have any moons as well as rings. Ceres is the first dwarf planet which is visited by NASA's Dawn Mission, launched in 2015, (Dawn was the very first spacecraft to visit Vesta and Ceres) to study its surface, composition and history. Scientists would like to search for possible signs of life in Ceres. It has something many other planets don't, especially water R. S. Park et al. (2016)

\section{(ii) 2 Pallas}

2 Pallas is the second asteroid to be discovered and the third largest asteroid in the asteroid belt, by the German astronomer and physician Wilhelm Olbers on March 28,1802, following the discovery of Ceres. It is named after Pallas Athena, the Greek goddess of wisdom. Pallas mean distance from the sun of $2.77 \mathrm{AU}$ and orbital period of 4.62 years are typical for the asteroids situated between the orbits of Mars and Jupiter.

Pallas is an ellipsoidal shape which is an equivalent to a sphere with a diameter of $513 \mathrm{~km}$ (i.e.) about $15 \%$ of the diameter of the moon. Pallas's albedo is 0.15 and turns once on its axis every 7.8 hours. Now close-up images of Pallas from the Hubble space telescope unveil that the Pallas appear to be a protoplanet (Protoplanet are small celestial objects that are the size of the moon or a little bigger. They are small planets, even smaller version of a dwarf planet. Astronomers believe that these objects form during the creation of a solar system). Visible of the Pallas in the Hubble images were areas of dark and light on Pallas surface, showing that the water rich body might have gone through an internal change in the same way planets do. For the very first time, a huge depression was also seen on the Pallas. They were unable to find out if it was a crater, but the depression did argue something else important that it could have led to Pallas small family of asteroids orbiting in space. Pallas massive body is unique and its orbit is so much different from other asteroids. Because it is highly inclined.

\section{(iii) 3 Juno}

3 Juno was discovered by Karl Ludwig Harding on 


\section{International Journal of Engineering Applied Sciences and Technology, 2019 Vol. 4, Issue 8, ISSN No. 2455-2143, Pages 89-93 \\ Published Online December 2019 in IJEAST (http://www.ijeast.com)}

the night of Sep 1st, using a small $5 \mathrm{~cm}$ refracting telescope at the Lilienthal Observatory in Bremen, Germany. The Roman goddess Juno was the protector and the counsellor of the state and hele watch over women. The Greek goddess equivalent was Hera. The asteroid is far too small to show a visible disk, instead watch for its movement against the starry background on successive nights. Juno has an orbital period of 4.36 years is $233.92 \mathrm{~km}$ in diameter and has a rotational period of 7.21 hours. It has a relatively high surface reflecting of $-4 \%$ brighter than most stony chondrite type asteroids. Juno is the asteroid of covenants in relationships and between soul colleagues of bonds, agreements and commitments in relationships. Juno is not only of a personal relation, but also those of a business and economic nature to those of political leaders and nations of the world. Juno's day is February 14 our Valentine's day during which ceremony and celebrations are done for those of pure love.

\section{(iv) 4 Vesta}

Vesta was discovered on March 29,1807 by the German Physician Heinrich Wilhelm Matthans Olbers. He named the asteroid for the Roman goddess of virtue at the suggestion of Carl Fredrich Gaum. Vesta is the second largest of the asteroids exceeded Ceres only by measuring some $530 \mathrm{~km}$ in diameter and has an orbital period of 3.63 years. Gaffey, M.J., (1997). Vesta is a specially interesting asteroid in that it seems to have been differentiated into layers like the terrestrial planets. This entails some internal heat source in additional to the heat delivered by long-lived radio isotopes which alone would be inadequate to melt such a small object. There is also a massive impact basin so deep that it uncovers the mantle beneath Vesta's outer crust. It is the brightest asteroid in the sky, Vesta is sometimes visible from the Earth with the naked eyes. Vesta is believed to be the only remaining rocky protoplanet which likely coalesced to form the rocky terrestrial planets. Unlike most asteroids, the interior of Vesta is differentiated. Like the other terrestrial planets, the asteroid has cooled lava as a crust covering a rocky matter made of iron and nickel core. Duffard, R \& Carvano, J. (2004).

NASA's DAWN was the first spacecraft to be launched in September 2007 and entered the orbit around Vesta in later July 2011 bringing us breath taking images of vesta. Previous missions have revealed us a handful of asteroids but no larger than this hulking relic of the early solar system. Measuring 350 miles across and containing nearly $10 \%$ of the mass of the whole asteroid belt, vesta is a world unto itself. In October 2010, the Hubble Space Telescope photographed Vesta again. The resulting data showed that the asteroid was tilted roughly four degrees more than scientists originally assumed. These discoveries helped NASA to place the spacecraft in the suitable polar orbit around the asteroid. DAWN requires sunlight in order to perform assignments like mapping and imaging.

\section{G. LATEST UPDATES}

The oldest impact creator on earth is also the greatest. Vredefort Crater in South Africa also called the Vredefort dome was originally $300 \mathrm{~km}$ across as by the scientists' estimates. A meteorite or asteroid larger than South Africa's Table Mountain flaming out the giant crater 2.02 billion years ago. According to NASA space rocks smaller than $25 \mathrm{~m}$ (82 ft.) will most likely burn up as they enter earth's atmosphere, which means that even if 2015TC25 hit earth, it probably wouldn't make it to the ground.

On February 2015, an asteroid ran into the atmosphere over the Russian city of Chelyabinsk, creating a shock wave that harmed 1200 people. The space rock is assumed to have measured about $65 \mathrm{ft}(20 \mathrm{~m})$ wide when it entered Earth's atmosphere. When an asteroid or part of it, rans into earth, it is called a meteorite. When asteroids come closer to earth, one of the most efficient ways to observe them is by using RADAR, such as the system at NASA's Goldstone Deep Space Communications Complex located in California. In September 2017, the near-earth asteroid 3122 Florence cruised by Earth at 4.4 million miles ( 7 million $\mathrm{km}$ ) or 18 times the distance to the moon. The flyby assured its size $(4.5 \mathrm{~km})$ and rotation period (2.4 hours) Sixty-six million years ago, dinosaurs had an ultimate bad day with a devastating asteroid collision, a reign that had lasted 180 million years was suddenly ended. The impact size known as the Chicxulub crater is centred on the Yucatan Peninsula in Mexico. The asteroid is thought to be 10 to $15 \mathrm{~km}$ wide, but the velocity of its collision caused the creation of a much larger crater, $150 \mathrm{~km}$ in diameter- the second largest crater on the planet. The crash that killed dinosaur threw huge amounts of debris into the air and produced massive tidal waves to consume the parts of the American Continents. There is also proof of substantial fires from that point in history. Were it not for the asteroid, dinosaurs might have survived a little longer although with modern birds, Eric Hand (2015), mammals and reptiles starting to develop, they may not have dominated as they once did. Asteroid warning space rock comparable to dinosaur killer is heading Earth's way by Sean Martin, Aug 16, 2019.

The asteroid known as 1990 MU at present is accomplishing another orbit of sun and in 2027 it could come daringly close to Earth. Asteroid $1990 \mathrm{MU}$ is about 4 to 9 kilometers in diameter and on June 6, 2027, it is predicted to come in less than 0.03 AU (Astronomical Unit). One $\mathrm{AU}$ is the distance between the Earth and the Sun, therefore coming in less than just $0.03 \mathrm{AU}$ is daringly close.

In January 2017, NASA selected two projects, Lucy and Psyche, using its discovery program and has Planned to launch in October 2021, Lucy will visit the object in the asteroid belt before going on to study six Trojan asteroids. Psyche will travel to 16 Psyche, a huge metallic asteroid that may be the bosom of a former Mars-size planet, plundered of its crust through violent collisions. NASA has begun to work on plans for its own asteroid-capture mission. Still we are getting more information about asteroids and their influence on earth and 


\section{International Journal of Engineering Applied Sciences and Technology, 2019 \\ Vol. 4, Issue 8, ISSN No. 2455-2143, Pages 89-93 \\ Published Online December 2019 in IJEAST (http://www.ijeast.com)}

other planets through latest updates by all sky gazers.

\section{CONCLUSION}

The mystery of the asteroids is myth in the creation of God. Even though asteroids are not having certain orbit but it would not often depart from their residence. The most dangerous asteroids are extremely rare, according to NASA. As asteroid capable of global destruction would have to be wider than a quarter mile. NASA officials say that on average asteroids would largely strike earth only once in every 1000 centuries. Smaller asteroids that may strike earth every 1000 to 10,000 years could demolish a city or cause cataclysmic tsunamis. Though many asteroids are skimming the earth orbit they have not affected brutally.

\section{REFERENCE}

[1] Gradie, J., Veverka, J. The composition of the Trojan asteroids. Nature 283, 840-842 (1980).

[2] Zellner, B. in Asteroids (ed. Gehreis, T.) 783-808 (University of Arizona Press, Tucson, 1979).

[3] Edward A. Cloutis, Matthew R.M. Izawa and Pierre Beck, Reflectance Spectroscopy of Chondrites, Primitive Meteorites and Asteroids, 10.1016/B978-0-12-8133255.00004-5, (273-343), (2018).

[4] Duffard, R., Lazzaro, D., Licandro, J., Sanctis, M., Capria, M., \& Carvano, J. (2004). Mineralogical characterization of some basaltic asteroids in the neighborhood of (4) Vesta: First results. Icarus, 171, 120132.

[5] Gaffey, M.J., 1997. Surface lithologic heterogeneity of Asteroid 4 Vesta. Icarus 127, 130-157.

[6] Data accessed from https://geology.com/articles/nearearth-asteroids.shtml.

[7] Bobrovnikoff,N.T. 1931 Journal: Publications of the Astronomical Society of the Pacific, Vol. 43, No. 255, p.324 19

[8] R. S. Park et al. "A Partially Differentiated Interior for (1) Ceres Deduced from its Gravity Field and Shape," Nature, Vol. 537, 22 September 2016, p. 515..

[9] Eric Hand, "Comet Close-up Reveals a World of Surprises," Science, Vol. 347, 23 January 2015, p. 358..

[10] Derek C. Richardson, "Giants in the Asteroid Belt," Nature, Vol. 411, 21 June 2001, p. 899..

[11] Retrived from Asteroids: Fun Facts and Information About Asteroids By Charles Q. Choi September 20, 2017 Science \& Astronomy

[12] Data accessed from mailonline By Joe Pinkstone For Mailonline Published: 10:50 GMT, 24 December 2018
[13] Retrived from advocator.ca/science/asteroid-doomsdaymassive-space-rock-similar-to-the-one-that-killeddinosaurs-is-heading-our-way/12049.

[14] Roig, F., Nesvorny, D., Gil-Hutton, R., \& Lazzaro, D. (2007). V-type Asteroids in the middle Main Belt. Icarus 194(1), 124-136. 